\title{
Sensibilidade antimicrobiana de bactérias aeróbicas isoladas de úlceras leishmanióticas, em Corte de Pedra, BA
}

\author{
Antimicrobial susceptibility of aerobic bacteria isolated from \\ leishmaniotic ulcers in Corte de Pedra, BA
}

\author{
Luis Angel Vera ${ }^{1}$, Jefferson Lessa Soares de Macedo ${ }^{1}$, Isolina Allen Ciuffo ${ }^{2}$, \\ Conceição Guerra Santos ${ }^{2}$ e João Barberino Santos ${ }^{1}$
}

\begin{abstract}
RESUMO
Um estudo prospectivo, sobre a sensibilidade antimicrobiana da flora bacteriana em úlceras cutâneas leishmanióticas, foi realizado em pacientes portadores de leishmaniose tegumentar, em Corte de Pedra, Bahia. Foram estudados 84 pacientes, principalmente adolescentes e adultos dedicados à lavoura, apresentando lesão cutânea única. Staphylococcus aureus predominou (83\%) nas culturas, sendo sensível à maioria dos antibióticos testados. Flora bacteriana mista esteve presente na úlcera em 37 (44,1\%) pacientes. Entre as bactérias Gram-negativas isoladas, foram mais freqüentes Enterobacter sp (13,1\%), Proteus sp (8,3\%), Pseudomonas aeruginosa (7,1\%) e Klebsiella sp (7,1\%), sendo sensíveis principalmente à ciprofloxacina, aminoglicosídeos, cefalosporinas de terceira geração e carbapenêmicos.
\end{abstract}

Palavras-chaves: Leishmaniose tegumentar. Infecção. Bactéria.

\begin{abstract}
A prospective study regarding aspects of antimicrobial susceptibility aspects was realized among patients with tegumentary leishmaniasis in Corte de Pedra, Bahia. Cases were composed mainly of adolescent and adult farmer patients with single lesions. Staphylococcus aureus predominated (83\%) in the culteres with susceptibility to the majority of antibiotics. A mixed bacterial flora in ulcers was encountered in 37 (44,1\%) patients. Among the Gram-negative bacteria isolated, Enterobacter sp (13.1\%), Proteus sp (8.3\%), Pseudomonas aeruginosa (7.1\%) and Klebsiella sp (7.1\%) were mainly found with susceptibility to ciprofloxacin, aminoglycosides, third generation cephalosporin and carbapenems.
\end{abstract}

Key-words: Tegumentary leishmaniasis. Infection. Bacteria.

A lesão cutânea ulcerada, manifestação clínica mais freqüente da leishmaniose tegumentar, geralmente localizada em membros inferiores, permanece ativa durante meses, expondo-se à colonização por microorganismos, componentes ou não da flora normal da pele. As condições de calor, umidade e má higiene, que acontecem frequientemente em ambiente rural nas regiões tropicais, associadas à proximidade dessas úlceras com o solo, favorecem, sobremodo, a colonização por diversas bactérias ${ }^{20}$.

Existem poucos relatos sobre a prevalência de bactérias em úlceras leishmanióticas. Em Corte de Pedra $(\mathrm{BA})^{20}$, encontrou-se infecção secundária em 54,2\% das úlceras estudadas, sendo mais comum $S$. aureus, semelhante aos estudos realizados no Irã ${ }^{9}$ e no Estado do Maranhão, Brasil ${ }^{16}$.
O objetivo desse estudo foi avaliar a sensibilidade antimicrobiana das bactérias isoladas em úlceras de pacientes com leishmaniose cutânea, em Corte de Pedra, Bahia, Brasil.

\section{PACIENTES E MÉTODOS}

O estudo compreendeu pacientes que procuraram espontaneamente o Centro de Saúde de Corte de Pedra, área endêmica de leishmaniose tegumentar, no período de agosto a dezembro de 1998.

A metodologia empregada para o diagnóstico de leishmaniose e isolamento das bactérias nas úlceras já foi

\footnotetext{
1. Núcleo de Medicina Tropical e Nutrição da Universidade de Brasília, Brasília, DF. 2. Laboratório Central (LACEN) de Salvador, BA, Brasil. Endereço para correspondência: Dr. Jefferson Lessa Soares de Macedo. SQS 213, Bloco H, Apt ${ }^{0}$ 104, Asa Sul, $70292-080$ Brasília, DF. Telefax: $55613327-8415$

e-mail: jlsmacedo@yahoo.com.br

Recebido para publicação em 31/8/2004

Aceito em 29/10/2005
} 
anteriormente descrita e, os critérios de exclusão abrangeram, entre outros, o uso de antimicrobiano sistêmico nas 48 horas anteriores à admissão (ou de penicilina benzatina nas últimas três semanas) ${ }^{20}$. Não foram incluídas as bactérias isoladas que não apresentaram antibiograma.

A sensibilidade antimicrobiana foi avaliada pelo método de difusão em placa (teste de Kirby-Bauer), empregando-se discos de antimicrobianos (Cecon $®$ ) específicos para bactérias Grampositivas e Gram-negativas. Os antimicrobianos usados na avaliação da sensibilidade são apresentados nas Tabelas 2 e 3. Após coleta de material da úlcera com $s w a b$, todos pacientes iniciaram tratamento com antimoniato-N-metilglucamina $\mathrm{EV}$, $15-20 \mathrm{mg} / \mathrm{Sb}^{\mathrm{v}} / \mathrm{kg} / \mathrm{dia}$, durante 20 dias consecutivos e, lavagem da úlcera com água e sabão duas vezes ao dia, durante 15 dias.

\section{RESULTADOS}

Foram incluídos 84 pacientes, com a média de idade de 26 anos (variação de 4 a 72 anos), sendo 67,9\% do gênero masculino. Os lavradores representaram $60,7 \%$ da amostra. A freqüência de localização da úlcera mais antiga foi: abaixo dos joelhos 40 (47,6\%), membros superiores 16 (19\%), cabeça $11(13,1 \%)$, tronco $10(11,9 \%)$ e coxas $7(8,3 \%)$.

0 uso de antimicrobiano sistêmico, anterior à inclusão no estudo, foi relatado por $44 \%$ dos pacientes: penicilinas $(23,8 \%)$, tetraciclinas $(11,9 \%)$, tetraciclina + penicilina $(5,9 \%)$ e eritromicina + penicilina (2,4\%). Vários tipos de tratamentos locais das lesões, anterior à inclusão no estudo, foram relatados por 66 (78,6\%) pacientes, incluindo o uso de antimicrobianos tópicos (tetraciclinas e penicillinas), antissépticos (merthiolate/ mercúrio) e emplastros de plantas, tais como a Astronium lecointei Ducke (aroeira), a Anacardium occidentale L. (cajueiro), a Chenopodium ambrosioides L. (mastruz) e Jatropha gossypifolia L. (pinhão roxo).

No momento da admissão, foi encontrada secreção na úlcera estudada em 71 (84,5\%) pacientes: secreção melisérica em 33 (39,3\%) e purulenta em 38 (45,2\%). Evidenciou-se odor fétido na úlcera em quatro $(4,8 \%)$ pacientes e sinais inflamatórios perilesionais em três (3,6\%).

Os três pacientes com sinais inflamatórios perilesionais foram considerados com infecção secundária aparente e tratados com sulfametoxazol/trimetoprima na dose de 1 comprimido de 160/800mg de 12/12 horas durante 1 semana; além da terapêutica específica com antimoniato$\mathrm{N}$-metilglucamina IV,15-20mg/Sb/ $/ \mathrm{Kg} / \mathrm{dia}$ de, durante 20 dias consecutivos; e foi recomendado a lavagem da úlcera cutânea com água e sabão comum 2 vezes ao dia, durante 15 dias.

A presença de linfadenomegalia regional satélite foi observada em 73 (86,9\%) pacientes. Contudo, a temperatura axilar aferida antes do início do tratamento específico foi, em média, $36,8^{\circ} \mathrm{C}( \pm 0,5)$, com variação de 35,8 a $37,9^{\circ} \mathrm{C}$.

0 tempo de evolução da úlcera mais antiga foi, em média, 6,9 ( \pm 3) semanas, com variação de 2 a 20 semanas.
As culturas bacteriológicas do material da lesão leishmaniótica mais antiga, obtido mediante $s w a b$ da base da úlcera, mostraram evidência de bactérias Gram-positivas em 35 (41,6\%) pacientes, bactérias Gram-negativas 12 $(14,3 \%)$ e flora mista em 37 (44,1\%). Quanto à flora mista, as associações mais freqüentes foram, respectivamente: a) $S$. aureus e Pseudomonas aeruginosa $(13,2 \%)$; b) $S$. aureus e Enterobactersp $(11,3 \%)$; c) $S$. aureus e Proteus sp (9,4\%); d) $S$. aureus e Streptococcus sp (9,4\%).

Dentre as bactérias Gram-positivas, a mais freqüente foi Staphylococcus aureus $(83,3 \%)$ e, entre as Gram-negativas, a mais isolada foi Enterobacter sp (13,1\%) (Tabela 1). S. aureus apresentou sensibilidade antimicrobiana acima de $90 \%$ à amoxicilina/clavulanato, cefalotina, gentamicina, ciproflaxacina, clindamicina, sulfametoxazol/trimetoprima, oxacilina e vancomicina e, abaixo de 80\%, à tetraciclina e à eritromicina (Tabela 2).

Tabela 1 - Frequiência de bactérias isoladas de úlceras leishmanióticas em 84 pacientes tratados em Corte de Pedra, Bahia, no período de agosto a dezembro de 1998.

\begin{tabular}{lcr}
\hline & \multicolumn{2}{c}{ Frequiência } \\
\cline { 2 - 3 } Bactéria isolada & $\mathrm{n}^{0}$ & $\%$ \\
\hline Staphylococcus aureus & 70 & 83,3 \\
Enterobacter sp & 11 & 13,1 \\
Staphylococcus epidermidis & 10 & 11,9 \\
Streptococcus sp & 7 & 8,3 \\
Proteus sp & 7 & 8,3 \\
Pseudomonas aeruginosa & 6 & 7,1 \\
Klebsiella sp & 6 & 7,1 \\
Escherichia coli & 5 & 5,9 \\
Serratia marcescens & 4 & 4,8 \\
Citrobacter freundii & 3 & 3,8 \\
\hline
\end{tabular}

Tabela 2 - Percentual de sensibilidade antimicrobiana de bactérias Grampositivas isoladas em 84 pacientes tratados em Corte de Pedra, Bahia, no período de agosto a dezembro de 1998.

\begin{tabular}{lccc}
\hline & \multicolumn{3}{c}{ Bactérias Gram-positivas } \\
\cline { 2 - 4 } & S. aureus & S. epidermidis & Streptococcus sp \\
& $\mathrm{n}^{0}=70$ & $\mathrm{n}^{0}=10$ & $\mathrm{n}^{0}=07$ \\
Antibióticos testados & $\%$ & $\%$ & $\%$ \\
\hline Amoxicilina/clavulanato & 95,7 & 100,0 & $\mathrm{NT}$ \\
Cefalotina & 95,7 & 100,0 & 100,0 \\
Gentamicina & 97,1 & 100,0 & 85,7 \\
Ciprofloxacina & 97,1 & 100,0 & 100,0 \\
Clindamicina & 94,3 & 100,0 & 100,0 \\
Tetraciclina & 75,7 & 100,0 & 0,0 \\
Eritromicina & 78,6 & 100,0 & 100,0 \\
SMT/TMP* & 94,3 & 100,0 & 85,7 \\
Vancomicina & 100,0 & 100,0 & $\mathrm{NT}$ \\
Oxacilina & 100,0 & 100,0 & 100,0 \\
\hline
\end{tabular}

* SMT/TMP = Sulfametoxazol/trimetoprima; NT = não testado

As bactérias Gram-negativas apresentaram sensibilidade acima de $70 \%$ à ciprofloxacina, aminoglicosídeos e imipenem. Entretanto, Enterobacter sp e P. aeruginosa, que foram as bactérias mais comumente associadas a $S$. aureus, apresentaram baixa sensibilidade ao sulfametoxazol/ trimetoprima, cefalotina e cefoxitina (Tabela 3). 
Tabela 3 - Percentual de sensibilidade antimicrobiana de bactérias Gram-negativas isoladas em 84 pacientes tratados em Corte de Pedra, Bahia, no período de agosto a dezembro de 1998.

\begin{tabular}{|c|c|c|c|c|c|c|c|}
\hline \multirow{4}{*}{$\begin{array}{l}\text { Antibióticos } \\
\text { testados }\end{array}$} & \multicolumn{7}{|c|}{ Bactérias Gram negativas } \\
\hline & Enterobactersp & Proteus sp & $P$. aeruginosa & Klebsiella sp & E. coli & S. marcecens & C. freundii \\
\hline & $\mathrm{n}^{\underline{0}}=11$ & $\mathrm{n}^{0}=7$ & $\mathrm{n}^{0}=6$ & $\mathrm{n}^{0}=6$ & $\mathrm{n}^{0}=5$ & $\mathrm{n}^{0}=4$ & $\mathrm{n}^{0}=3$ \\
\hline & $\%$ & $\%$ & $\%$ & $\%$ & $\%$ & $\%$ & $\%$ \\
\hline SMT/TMP* & 54,5 & 100,0 & 16,7 & 83,3 & 60,0 & 100,0 & 100,0 \\
\hline Cefalotina & 9,1 & 57,1 & 0,0 & 83,3 & 60,0 & 0,0 & 33,3 \\
\hline Cefoxitina & 18,2 & 57,1 & 0,0 & 83,3 & 100,0 & 25,0 & 66,6 \\
\hline Ceftriaxona & NT & 100,0 & 50,0 & 100,0 & 100,0 & 100,0 & 100,0 \\
\hline Ciprofloxacina & 100,0 & 100,0 & 100,0 & 100,0 & 100,0 & 100,0 & 100,0 \\
\hline Gentamicina & 72,7 & 100,0 & 100,0 & 100,0 & 100,0 & 100,0 & 100,0 \\
\hline Imipenem & 100,0 & 100,0 & 100,0 & 100,0 & 100,0 & 100,0 & 100,0 \\
\hline Amicacina & 100,0 & 100,0 & 100,0 & 100,0 & 100,0 & 100,0 & 100,0 \\
\hline
\end{tabular}

\section{DISCUSSÃo}

As características epidemiológicas e clínicas dos pacientes avaliados são representativas da população atingida pela leishmaniose tegumentar produzida por $L$. (V.) braziliensis na região do estudo ${ }^{13}$.

0 predomínio de adolescentes e de adultos do gênero masculino dedicados à lavoura, concorda com a principal maneira de aquisição da leishmaniose tegumentar, considerada doença ocupacional. Além disso, a ocorrência mais freqüente de lesão única, geralmente abaixo dos joelhos, coincide com outras casuísticas da região de estudo ${ }^{613}$. A linfoadenopatia é um achado comum em pacientes não tratados com leishmaniose cutânea recente; nesse estudo, foi documentada em $86,9 \%$ dos casos. 0 acometimento linfonodal deve-se primariamente à presença da $L$. braziliensis e, secundariamente, à colonização ou infecção bacteriana da úlcera 3 .

A bactéria mais prevalente nas úlceras leishmanióticas foi $S$. aureus, como em outros estudos realizados no Brasil ${ }^{16}$, Equador ${ }^{5}$ e Irã ${ }^{9}$. A conduta terapêutica inicial nas úlceras leishmanióticas cutâneas consiste no uso local de água e sabão durante 15 dias, concomitante ao tratamento com o antimonial pentavalente. Em caso de sinais inflamatórios perilesionais, além dos limites da úlcera, suspeita-se de infecção secundária, dessa forma estaria indicado antibioticoterapia oral com sulfametoxazol/trimetoprima como primeira escolha, devido ao baixo custo e à atividade antimicrobiana acima de $85 \%$ contra $S$. aureus, S. epidermidis, Streptococcus sp, Proteus sp, Serratia marcecens e Citrobacter freundii. Entretanto, a ciprofloxacina estaria indicada como segunda escolha, pois apresenta $97,1 \%$ e $100 \%$ de atividade antimicrobiana contra $S$. aureus e bactérias Gram-negativas, respectivamente. Entretanto, a ocorrência de infecção bacteriana secundária aparente ao exame clínico, ou seja, a presença de sinais inflamatórios perilesionais, além dos limites da úlcera, é rara. A ocorrência nesse estudo foi 3,6\% (3 casos). 0 baixo índice de infecção bacteriana secundária indica que as bactérias isoladas agem como microrganismos colonizantes nas úlceras. A colonização bacteriana pode ter um papel importante no aparecimento de infecção da ferida e da sepse em pacientes imunocomprometidos ${ }^{14}$.
No Irã, Edrissian e cols ${ }^{9}$ verificaram sensibilidade de $96,9 \%$ de $S$. aureus, isolado de úlceras leishmanióticas, à eritromicina. A eritromicina é o antimicrobiano de escolha no tratamento das infecções bacterianas dessas úlceras naquele país. Entretanto, em Corte de Pedra, Bahia, foi verificado uma sensibilidade abaixo de $80 \%$ do $S$. aureus à tetraciclina e à eritromicina. Essa menor sensibilidade poderia ser devida ao uso de antimicrobianos sistêmicos ou tópicos, inclusive tetraciclina e eritromicina, anterior às 48 horas de inclusão no estudo, além de antissépticos e emplastros de plantas com atividade antimicrobiana. 0 uso do extrato de Anacardium occidentale (cajueiro) é o tratamento fitoterápico mais utilizado na região do estudo, inclusive com elevada atividade in vitro contra promastigotas de $L$. braziliensis ${ }^{10}$, além disso, tem boa atividade antimicrobiana in vitro contra bactérias Gram-positivas, Escherichia coli e Pseudomonas aeruginosa ${ }^{2}{ }^{11}$. Atividade antimicrobiana, inclusive contra micobactérias, tem sido demonstrada em plantas dos gêneros Jatropha (pinhão) ${ }^{1}$, Chenopodium (mastruz) ${ }^{12}$ e Astronium (aroeira) ${ }^{19}$. Plantas do gênero Astronium (aroeira) também apresentam atividade antiinflamatória, sendo utilizadas na região contra tosse, bronquite, inflamação ovariana e úlceras externas ${ }^{818}$. Além disso, as bactérias que são resistentes aos antissépticos apresentam alta resistência a antimicrobianos ${ }^{4}{ }^{47}$. Portanto, o uso prévio de antimicrobianos, antisséticos e emplastros de plantas pode ter contribuído para seleção da flora bacteriana e justificar a percentagem de $44,1 \%$ de flora mista presente nas lesões dos pacientes estudados.

Não está bem claro o papel ou as consequiências dessa flora microbiana na evolução das úlceras leishmanióticas ${ }^{15}$. Um estudo aponta que cepas de $S$. aureus mortas ou componentes da membrana celular da bactéria, na presença de interferon-gama, aumentam a atividade leishmanicida de macrófagos in vitro ${ }^{7}$. Tem sido observado maior percentual de infecção bacteriana secundária em pacientes com suspeita de leishmaniose cutânea e exame parasitológico negativo 9 .

Devido ao baixo índice de sinais inflamatórios perilesionais, indicando mais colonização do que infecção, o manejo tópico das úlceras leishmanióticas deve ser feito com água e sabão, reservando-se o uso de antimicrobiano aos raros casos de infecção patente; sulfametoxazol/trimetoprima seria a primeira escolha e, a ciprofloxacina, a escolha secundária. 


\section{REFERÊNCIAS BIBLIOGRÁFICAS}

1. Aiyelaagbe 00. Antibacterial activity of Jatropha multifida roots. Fitoterapia 72: 544-546, 2001.

2. Akinpelu DA. Antimicrobial activity of Anacardium occidentale bark. Fitoterapia 72: 286-287, 2001.

3. Barral A, Guerreiro J, Bomfim G, Correia D, Barral-Netto M, Carvalho EM. Lymphadenopathy as the first sign of human cutaneous infection by Leishmania braziliensis. The American Journal of Tropical Medicine and Hygiene. 53: 256-259, 1995.

4. Cervantes-Vega C, Chavez J. Susceptibility to mercurials of clinical Pseudomonas aeruginosa isolated in México. Antonie Van Leeuwenhoek 53: 253-259, 1987.

5. Coronel VV, Martini L, Alava JJ, Garcia NT, Gomez EA, Hashiguchi Y. Bacterial flora in suspected Leishmania ulcers of pacients from an endemic focus on the Pacific coast of Ecuador. In: Hashiguchi Y (ed) Studies on New World leishmaniasis and its transmission, with particular reference to Ecuador. Research Report Series No. 3, Kyowa Printing \& Co, Kochi, p. 125-126, 1992

6. Costa JML, Osako NK, Vale KC, Lago EL, França F, Vexenat JA, Marsden PD. Ocorrência familiar da leishmaniose tegumentar americana em uma região endêmica, Corte de Pedra, Bahia. Revista da Sociedade Brasileira de Medicina Tropical 19: 197-198, 1986

7. Cunha FQ, Moss DW, Leal LM, Moncada S, Liew FY. Induction of macrophage parasiticidal activity by Staphylococcus aureus and exotoxins through the nitric oxide synthesis pathway. Immunology 78: 563-567, 1993.

8. Desmarchelier C, Romão RL, Coussio J, Ciccia G. Antioxidant and free radical scavenging activities in extracts from medicinal trees used in the 'Caatinga' region in northeastern Brazil. Journal of Ethnopharmacology 67: 69-77, 1999

9. Edrissian GH, Mohammadi M, Kanani A, Afshar A, Hafezi R, Ghorbani M, Gharagozloo AR. Bacterial infections in suspected cutaneous leishmaniasis lesions. Bulletin of the World Health Organization 68: 473-477, 1990.

10. França F, Lago EL, Marsden PD. Plants used in the treatment of leishmanial ulcers due to Leishmania (Viannia) braziliensis in an endemic area of
Bahia, Brazil. Revista da Sociedade Brasileira de Medicina Tropical 29: 229-232, 1996

11. Kudi AC, Umoh JU, Eduvie LO, Gefu J. Screening of some Nigerian medicinal plants for antibacterial activity. Journal of Ethnopharmacology 67: 225-228, 1999

12. Lall N, Meyer JJM. In vitro inhibition of drug-resistant and drug-sensitive strains of Mycobacterium tuberculosis by ethnobotanically selected South African plants. Journal of Ethnopharmacology 66: 347-354, 1999.

13. Llanos-Cuentas EA, Marsden PD, Lago EL, Barreto AC, Cuba CC, Johnson WD. Human mucocutaneous leishmaniasis in Três Braços, Bahia - Brazil. An area of Leishmania braziliensis transmission. II Cutaneous disease. Presentation and evolution. Revista da Sociedade Brasileira de Medicina Tropical 17: 169-177, 1984.

14. Macedo JLS, Rosa SC, Castro C. Sepsis in burned patients. Revista da Sociedade Brasileira de Medicina Tropical 36: 647-652, 2003.

15. Oliveira MR, Tafuri WL, Nicoli JR, Vieira EC, Melo MN, Vieira LQ. Influence of microbiota in experimental cutaneous leishmaniasis in Swiss mice. Rev Inst Med Trop São Paulo 41: 87-94, 1999.

16. Pereira ALN, Cella WP, Oliveira EG, Moreira IV, Filho SAR, Gonçalves EGR, Costa JML. Infecção secundária em leishmaniose tegumentar americana: perfil bacteriano e sensibilidade a antibióticos. Revista da Sociedade Brasileira de Medicina Tropical 32 (supl 1): 223, 1999.

17. Porter FD, Silver S, Ong C, Nakahara H. Selection for mercurial resistance in hospital settings. Antimicrobial, Agents and Chemotherapy 22: 852-858, 1982.

18. Rao VS, Viana GSB, Menezes AMS, Gadelha MGT. Studies on the antiulcerogenic activity of Astronium urundeuva Engl. II. Aqueous extract. Brazilian Journal of Medical and Biological Research 20: 803-805, 1987.

19. Salvat A, Antonacci L, Fortunato RH, Suarez EY, Godoy HM. Antimicrobial activity in methanolic extracts of several plant species from northern Argentina. Phytomedicine 11: 230-234, 2004.

20. Vera LA, Santos JB, Macedo VO, Magalhães AV, Ciuffo IA, Santos CG. Avaliação da influência da infecção bacteriana secundária na evolução da leishmaniose cutânea em Corte de Pedra, Bahia. Revista da Sociedade Brasileira de Medicina Tropical 34: 233-237, 2001 